\title{
Could mRNA Vaccine be Helping Promote SARS-CoV-2 Variant Emergence and Prolonging the COVID-19 Pandemic
}

\author{
Mohammed Khalid, $M D^{*}$
}

\begin{abstract}
Section of Pulmonary Medicine, Department of Medicine, King Faisal Specialist Hospital and Research Center, Saudi
\end{abstract} Arabia

COVID-19 vaccine brought a sigh of relief to COVID-19 affected world but as its use is increasing so are the emerging associated complications such as the systemic thrombosis and clot formation [1] and hypersensitivity reactions [2] are now well-known there will be long term complications which may emerge as more people get vaccinated and as the time passes since we humans are the experimental subjects. But one concern that is extremely bothersome if it is considered to be a valid concern than that may require the authorities to quickly evaluate the use of mRNA vaccine.

The concern is the mRNA vaccine consists of a single viral spike protein genetic information [3] the antibody generated in host reacts to a single viral spike protein by doing so it merely makes the virus ineffective but it does not kill the virus [4] maybe by doing that it encourages the virus to mutate and generate antibody resistant strains since it is a single protein antigen antibody formation that makes it easier for the virus to adapt and generate resistant strain to it. Countries that started vaccination earlier are already suffering from emergence of more rapidly transferable and deadly virus strains like India a front liner in vaccine use now a victim of dual mutant strain of virus [5] with very rapid spread of the virus the new cases are with increased severity of illness forcing a complete lockdown in many cities same issue true in US, UK and EU. Recent case reports of breakthrough infections in post vaccinated patients with two different variants highlights the same concern of emergence of resistant strains [6]. Could this limited mRNA vaccine antigen antibody reaction without addressing the whole viral genome the spike protein nucleocapsid and envelop protein may be making it easy for the virus to generate vaccine resistant strains causing a sustained relentless COVID-19 devastating pandemic. If what I believe is true that current technology of a single protein antigen antibody mRNA vaccine is helping generate new more lethal variants due to its limited target on virus spike protein helping virus to mutate and generate new resistant and more lethal strains than the mRNA vaccine may sustain COVID-19 disease indefinitely with worsening severity as time passes if this hypothesis is true, all mRNA vaccines should be re-evaluated for its use. WHO and other health authorities should look into this potential but a reasonable concern to prevent persistent COVID-19 pandemic.

The pharmaceutical companies should concentrate to make whole virus vaccine like traditional influenza vaccine or the way Chinese COVID-19 vaccine is made from whole dead virus proteins [7] which are more effective and current mRNA vaccine use should be evaluated by appropriate authorities on an urgent basis.

\section{References}

1. Schultz NH, Sørvoll IH, Michelsen AE, et al. (2021) Thrombosis and thrombocytopenia after ChAdOx1 $\mathrm{nCoV}-19$ vaccination. $\mathrm{N}$ Engl J Med 384: 22.

2. Blumenthal KG, Freeman EE, Saff RR, et al. (2021) Delayed Large Local Reactions to mRNA-1273 Vaccine against SARS-CoV-2. N Engl J Med 384: 1273-1277.

3. Samrat SK, Tharappel AM, Li Z, et al. (2020) Prospect of SARSCoV-2 spike protein: Potential role in vaccine and therapeutic development. Virus Res 288: 198141.

4. Wang $Z$, Schmidt $F$, Weisblum $Y$, et al. (2021) mRNA vaccineelicited antibodies to SARS-CoV-2 and circulating variants. Nature 592: 616-622.

5. Who (2021) New variant reported in India 16 April 2021.

6. Hacisuleyman E, Hale C, Saito $Y$, et al. (2021) Vaccine Breakthrough Infections with SARS-CoV-2 Variants. N Engl J Med 384: 2212-2218.

7. Kashte S, Gulbake A, El-Amin lii SF, et al. (2021) COVID-19 vaccines: Rapid development, implications, challenges and future prospects. Hum Cell 34: 711-733.

*Corresponding author: Mohammed Khalid, MD, Section of Pulmonary Medicine, Department of Medicine MBC 46, King Faisal Specialist Hospital and Research Center, PO Box 3354, Riyadh 11211, Saudi Arabia, Tel: 966-504181890

Accepted: September 09, 2021

Published online: September 11, 2021

Citation: Khalid M (2020) Could mRNA Vaccine be Helping Promote SARS-CoV-2 Variant Emergence and Prolonging the COVID-19 Pandemic. Vaccine Res Dev 2(1):8 